LOS was 8 days; $80 \%$ were discharged directly to home. In the majority of cases only 3 of the 5 acute management components were completed. More than $90 \%$ of patients received antibiotics but only one-third were prescribed guidelinedirected therapy. Intravenous steroids were used in the majority of cases, $67 \%$, in preference to oral steroids. On multivariate linear regression analysis adjusting for exacerbation severity, age, $\mathrm{FEV}_{1}$ and discharge destination, appropriate prescription of oral steroid therapy reduced LOS by 1.3 days, $\mathrm{p}=0.023$. By day $90,38 \%$ of patients had been readmitted to hospital. The probability of readmission was decreased in those who had received guideline-directed antibiotic therapy, OR 0.35 (95\% CI 0.15-0.79) $\mathrm{p}=0.012$. Adherence to acute COPD management guidelines is suboptimal. The greatest improvements in clinical outcomes were associated with prescription of oral steroids, where applicable, and guidelinedirected selection of antibiotic therapy. These components should, therefore, be a focus of strategies to improve quality of inpatient care in COPD.

\section{P66 EXACERBATION TELEMONITORING FOR COPD PATIENT UNDER LONG TERM OXYGEN THERAPY. STEP 1: BREATHING RATE MEASUREMENT VALIDATION}

${ }^{1,2}$ J Soler, ${ }^{3} \mathrm{XL}$ Le, ${ }^{3} \mathrm{DPQ}$ Nguyen, ${ }^{1,4} \mathrm{~L}$ Grassion, ${ }^{1} \mathrm{R}$ Antoine, ${ }^{1} \mathrm{~A}$ Guerder, ${ }^{1,5} \mathrm{~J}$ Gonzalez Bermejo. 'AP-HP, Groupe Hospitalier Pitié-Salpetrière Charles Foix, Service de Pneumologie et Réanimation Médicale (Département "R3S"), F-75013, Paris, France; ${ }^{2} \mathrm{CHU}$ de Nancy, Département de Pneumologie, Vandoeuvre-lès-Nancy, France; ${ }^{3}$ SRETT, BoulogneBillancourt, France; ${ }^{4}$ Service des Maladies Respiratoires, Hôpital du Haut Lévèque, Pessac, France; ${ }^{5}$ Sorbonne Université, UPMC Univ Paris 06, INSERM, UMRS 1158 Neurophysiologie respiratoire expérimentale et clinique, Paris, France
Introduction and Objectives Recent works shown that it was possible to predict COPD exacerbation based on monitoring of simple parameters, such as an increase of the breathing rate in spontaneous ventilation or under non-invasive ventilation. Continuous breathing rate monitoring of COPD patients could be, by consequence, a pertinent way to follow their state of health, or even to alert for exacerbation situation. We aim to validate the breathing rate measurement of a tele-monitoring solution (TeleOx, SRETT, Boulogne-Billancourt, France) on COPD patients under long term oxygen therapy, as a first step towards this perspective.

Methods Breathing rates of COPD patient under long term oxygen therapy were recorded over a night, simultaneously by TeleOx and by a reference polygraph (Nox T3, Nox Medical Inc. Reykjavik, Iceland). A median breathing rate was extracted every $5 \mathrm{~min}$ by TeleOx and compared to the exact same measurement from the reference polygraph. The agreement between the two methods is considered using PassingBablok regression on the measured breathing rate points set.

Results Passing-Bablok regression on 1099 measurement points coming from 14 representative patients, comparing Results from TeleOx and reference polygraph gives, within a confident interval of 95\%, a slope $b$ within $[0.976 ; 1.000]$ and an intercept $a$ within $[-0.217 ; 0.325]$.

Discussion The connected tele-monitoring device TeleOx, is capable of measuring the breathing rate of COPD subjects under long term oxygen therapy in excellent agreement with a reference polygraph. It opens encouraging e-medecin perspectives for this patient population.

10.1136/thoraxjnl-2017-210983.208

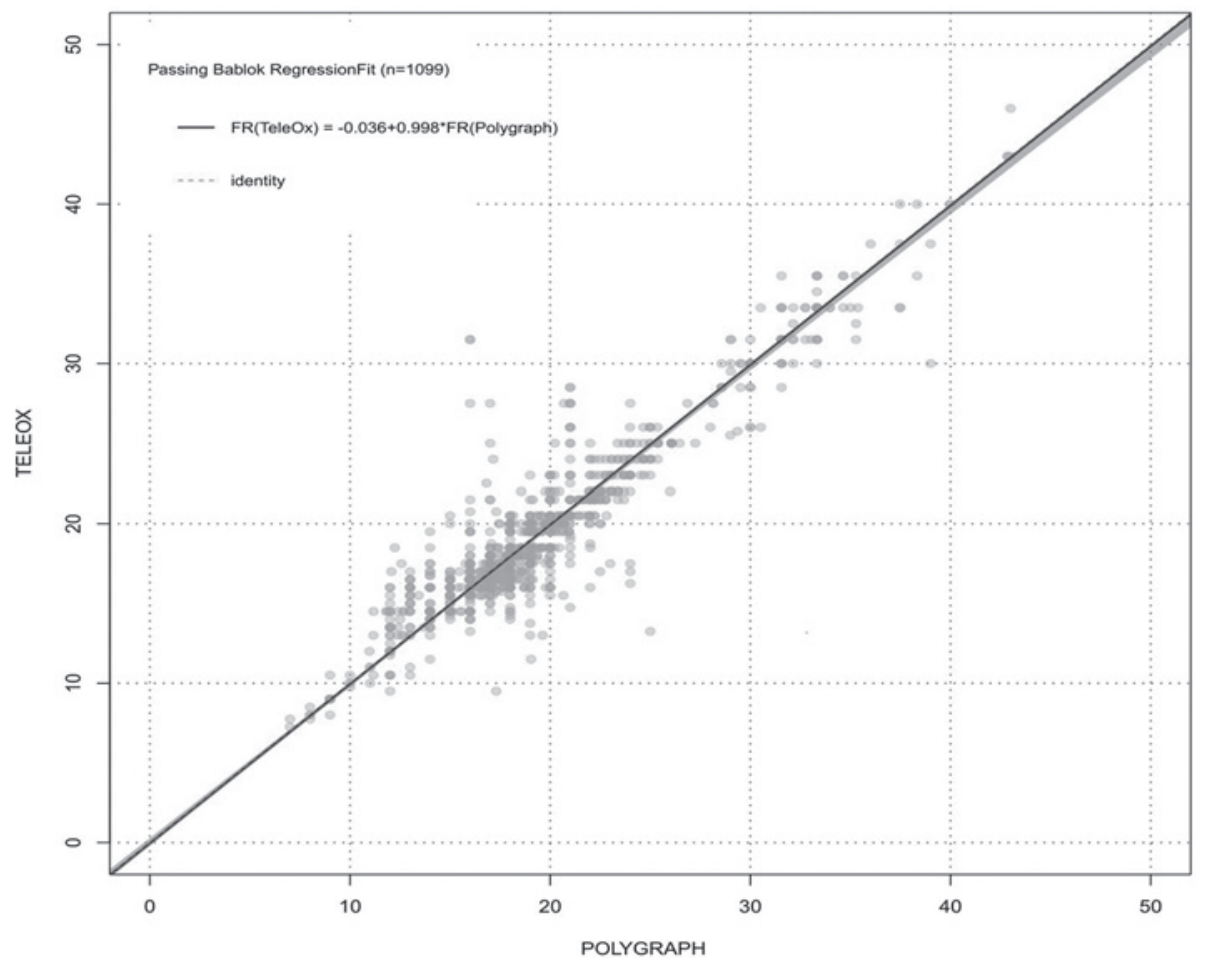

Abstract P66 Figure 1 Comparison of breathing rate (BR) measurement. Results by Tele0x and polygraph, using Passing-Bablok regression on a set of 1099 valid measurement points. BR measurements by TeleOx and polygraph are materialised in red points. Passing-Bablok regression line is presented in continuous blue line. Identity (red dashed line) and uncertainty envelop (blue) are presented as a guide for the lectors. 